\title{
Could a Cutaneous Lesion Mean Gastric Cancer? Case Report of a Rare Diagnostic Tool and the Management of a Cardiac Complication during Chemotherapy
}

\author{
Cátia Fava Gaspar, Maria João Leitão, Manuela Machado* \\ Medical Oncology Department, Portuguese Institute of Oncology of Porto (IPO Porto), Porto, Portugal \\ Email: *m.machado.fn@gmail.com
}

How to cite this paper: Caspar, C.F., Leitão, M.J. and Machado, M. (2020) Could a Cutaneous Lesion Mean Gastric Cancer? Case Report of a Rare Diagnostic Tool and the Management of a Cardiac Complication during Chemotherapy. Journal of Cancer Therapy, 11, 639-645.

https://doi.org/10.4236/jct.2020.1110054

Received: July 20, 2020

Accepted: October 24, 2020

Published: October 27, 2020

Copyright $\odot 2020$ by author(s) and Scientific Research Publishing Inc. This work is licensed under the Creative Commons Attribution International License (CC BY 4.0).

http://creativecommons.org/licenses/by/4.0/

\begin{abstract}
Cutaneous presentation of gastric cancer is very rare. This case report is about a patient with a metastatic gastric cancer in which the diagnosis was made by a cutaneous biopsy. Following this unusual diagnostic tool, the patient had an intracardiac thrombus, during the chemotherapy regimen, which wasn't a contraindication for pursuing chemotherapy treatment for over 12 months. This case report is presented to make clinicians aware of a possible atypical sign of presentation of gastrointestinal tract cancer, as well as the importance of clinical suspicion of a well-known problem affecting cancer patients such as venous thromboembolism and its repercussions in the treatment options.
\end{abstract}

\section{Keywords}

Gastric Cancer, Cutaneous Metastasis, Intracardiac Thrombus

\section{Introduction}

Gastric cancer is one of the five more common cancers in Portugal and one of the three more deadly cancers [1]. It is also proven that the northern region of Portugal has a higher incidence compared with regions of the country [2]. Around 35\% of gastric cancer patients present as metastatic [3], with the most common site of metastasis being the liver (48\%), peritoneum (32\%), lungs (15\%) and bone (12\%) [4].

Cutaneous metastasis and its manifestation of gastric cancer are rare and when it appears, it is usually associated with widespread disease and poor prog- 
nosis [5]. Although also rare, the only well-known skin sign of metastatic gastrointestinal cancer is Sister Mary Joseph's nodule, which also means advanced stage of malignancy and usually presents on the umbilicus [6].

Most gastric cancer patients with locally advanced/metastatic disease present with symptoms such as dyspepsia, dysphagia or abdominal pain, which is consistent with the most common sites of gastric cancer metastasis: lymph nodes, liver and peritoneum [5]. The incidence rate of cutaneous metastasis in this type of cancer is only about $0.8 \%-1 \%$ [7].

We present a case of a 56-year-old Portuguese man with a locally advanced gastric cancer presenting with a single cutaneous metastatic lesion, which is very rare. We also report, in the same patient, the development of an intracardiac thrombus and the challenging management of pursuing chemotherapy treatments.

\section{Case Report}

56-year-old male, with heavy past smoking habits (102 pack-years), known history of hypertension for 10 years, type II diabetes mellitus and family history of cancer (father and brother with pancreatic cancer at 60 and 58 years, respectively, and sister with breast cancer at 53 years).

The patient developed light progressive dyspeptic symptoms since the beginning of 2018 and symptomatic medication didn't solve the problem. It was then noted a cutaneous ulcerated anterior thoracic lesion, with $2.8 \times 1.4$ centimeters, which was removed in another institution. The pathology analysis revealed a probable metastatic adenocarcinoma, with signet ring cells and negativity for CK20 and TTF1, weak positivity for CK7 and strong positivity for CAM5.2 on the immunocytochemistry profile, suggesting a primary origin from digestive tract and pancreas, without exclusion of cutaneous annexes. The surgical margins were free of disease. The patient was then admitted at IPO Porto in August 2018. At this time, the analytic results showed an elevated tumor marker (ca 19.9 $108.1 \mathrm{U} / \mathrm{mL}$, with reference values until 37), favoring the gastrointestinal origin.

He was then subjected to further tests, including a thoracic-abdomen-pelvic computed tomography (CT) scan which showed a diffuse parietal thickening of the stomach with infiltrative atypia, with lymph nodes in the small curvature, dispersed within the remaining peri-gastric fat as well as mediastinal lymph nodes. The high endoscopy, a large ulcerated exophytic mass, with involvement of the cardia, anterior zone of the small curvature from the body to the incisura angularis, with an extension to the distal/of the esophagus was found. The pathology report of this mass confirmed the adenocarcinoma histology. Positron emission tomography (PET) scan confirmed the malignant neoplasia of the stomach and related the hypermetabolic lymph nodes as an inflammatory cause. The second look histology of the cutaneous lesions revealed a negative Her2 neoplasia.

Thus, since the cancer seemed localized and the patient had an excellent overall status with an Eastern Cooperative Oncology Group, Performance Status 
ECOG-PS 0, he started with a chemotherapy regimen of Cisplatin/5-Fluorouracilo in September 2018, with a posterior surgical intention.

After cycle 3 the patient presented at the oncology appointment with a history of cervical and facial oedema, with hypersudoresis, heart rate of 129 beats per minute and fainting sensation. Electrocardiogram revealed a sinus tachycardia with nonspecific alterations of the repolarization phase and prolonged QTc interval. He was then admitted as inpatient and the subsequent studies showed a massive bilateral pulmonary thromboembolism presumably associated with the central venous catheter (CVC) but with a subsequent migration of the thrombus to the right atrium (Figure 1), with $3.1 \times 1.8$ centimeters and with a systolic function lightly depressed. The patient started hypocoagulation with low molecular weight heparin, stopped the chemotherapy treatments for approximately 1 month and after that time was reevaluated by the Cardiology team. He denied any cardiovascular symptoms and the echocardiogram showed good left ventricular function, without any structural damages and apparent disappearance of the thrombus, so it was considered to have no contraindication to proceed treatments as planned.

At the reevaluation CT, the gastric diffuse thickening was visible (Figure 2) and the team found persistence of the thrombus at the superior vena cava with extension to the right atrium (Figure 3), possible disease progression at the pleural level and evidence of extensive peritoneal carcinomatosis. Since the patient was also clinically worse, with constipation, dysphagia for solid intake with occasional vomiting and worsened asthenia, it was admitted again for clinical optimization and further decisions regarding next steps of treatment.

During the hospital stay, he was again observed by the cardiology team and a new echocardiogram was performed confirming the inexistence of intracardiac thrombus and a preserved ejection fraction without signs of ischemia, endorsed with a myocardial perfusion imaging test (MIBI) which was also normal.

The patient was then considered safe to be treated with fluoropyrimidines and started palliative chemotherapy with a chemotherapy regimen-Folinic acid + Fluoracil + Irinotecan (FOLFIRI) in February 2019, always under inward surveillance for the first 7 cycles. From the $8^{\text {th }}$ cycle, the patient managed to do the treatments in ambulatory setting, until October 2019, completing a total of 18 cycles of the first line palliative chemotherapy regimen. After disease progression was documented on the response assessment CT scan, he was still fit for second line treatment and pursued chemotherapy with chemotherapy regimen of Folinic acid + Fluoracil + Oxaliplatin (FOLFOX) from November 2019 until March 2020. After that, the patient experienced clinical worsening until passing away in May 2020, 21 months after the diagnosis.

\section{Discussion}

Portugal has one of the highest gastric cancer mortality rates in Western Europe, particularly in the northern region [8] due to some well-known risk factors such as tobacco smoking, alcohol intake and diet high in processed meats [9]. 

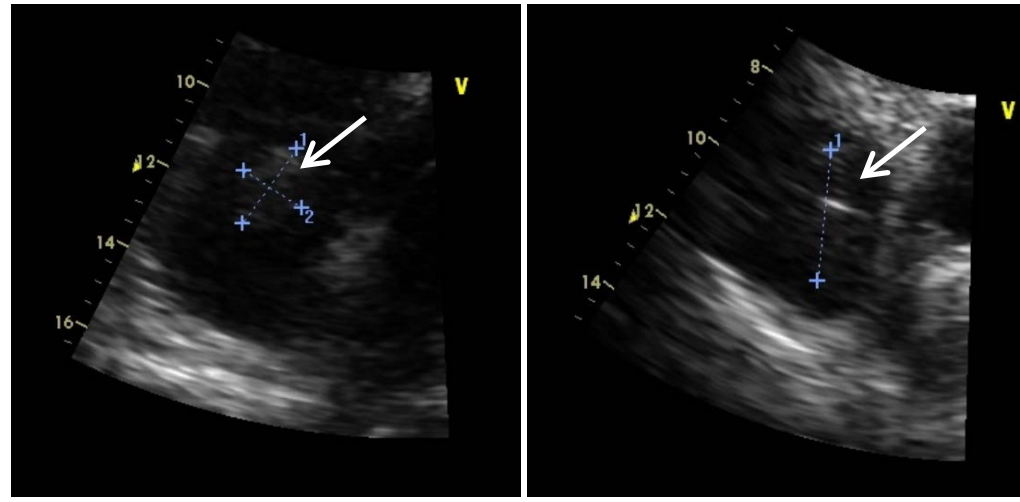

Figure 1. Intracardiac thrombus seen on echocardiogram.
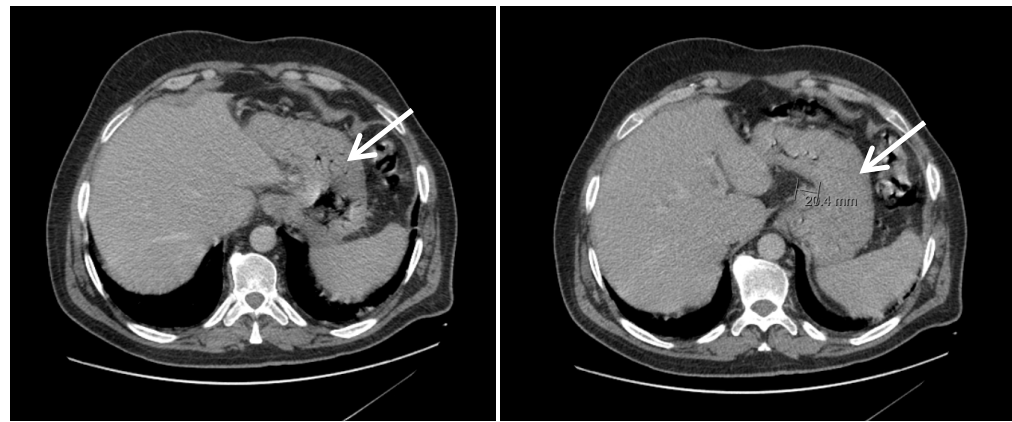

Figure 2. CT scan with diffuse gastric thickening after 3 cycles of chemotherapy (November/2018).

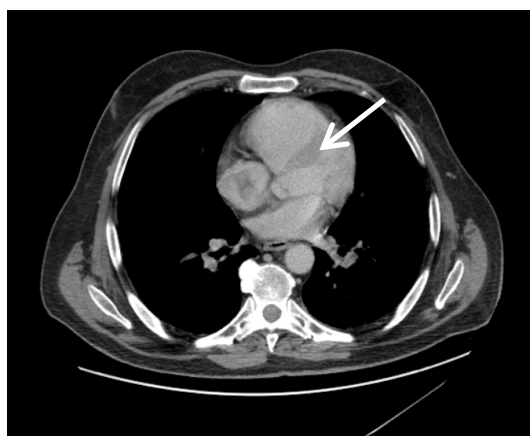

Figure 3. Same CT scan as presented above (November/2018) showing also and intracardiac thrombus.

This case exemplifies a very unusual presentation of a gastric cancer. Usually, cutaneous metastasis originate from the lung, breast, colon, melanoma, renal or head and neck cancer [10] [11]. It is known that spread of cancer, regardless of their origin, to a metastatic setting such as the skin, necessarily needs a hematogenous or lymphatic mechanism [11] but the rule is that the malignancy spreads in a stepwise manner, firstly from local metastasis and thereafter to a distant location [12]. This report case, besides representing a rare location of a metastatic site from a gastric cancer, is also peculiar since it was the only one single metastatic lesion at presentation, explaining why the patient was only lightly symptomatic. This reinforces the idea that accessible lesions such as skin lesion when 
suspicious, should always be biopsied since it is a way of getting the diagnosis in a short period of time, with minimum burden to the patient due to its non-invasive setting.

The fact that, after the diagnosis, the patient developed a cardiac complication could have prevented the continuation of treatment with further chemotherapy. Thrombosis is a well-known complication of oncological disease, cytotoxic therapy and intravascular devices, but the intracardiac extension on the thrombus is not usual.

Reports of intracardiac thrombus following cisplatin-based regimens and 5-FU, although rare, can be found in the literature [13] [14] [15], but very little is available on the safety of the continuation of treatment in these cases, as many patients don't survive or are not fit enough for therapy afterwards. On the case reported by Ikeda et al., the patient resumed therapy after resolution on the thrombus, which was not possible in our patient [16]. Kaul et al. reported a case in which the patient was severely symptomatic, justifying the need for surgical removal of the thrombus before adjuvant chemotherapy [17].

Our case report may provide evidence of safety in continuing therapy with careful vigilance and patient monitorization, when necessary.

Pursuing the palliative chemotherapy in this patient gave him an overall survival of 21 months with a good quality of life, despite the initial need for admission to the hospital every 2 weeks for the chemotherapy treatments at the beginning of the palliative care. Thus, comparing with the general prognosis of metastatic gastric tumors, known to be generally poor, with a median overall survival ranging from 4 to 12 months [18] [19] [20] our reported patient lived for a longer time, particularly because it was well supported by our institution even after severe cardiac complication-intracardiac thrombus-even when there were some doubts about its complete resolution, making it possible to make several treatments under active surveillance.

\section{Conclusion}

This case report is presented to make clinicians aware of one unusual sign such as a skin lesion may present as an initial manifestation of metastatic gastric cancer and to be noticed that, despite the metastatic setting at the diagnosis and even with a cardiac complication such as an intracardiac thrombus, some patients may still benefit, and for a long period of time, with chemotherapy regimens, when some effort of all the assistant team were made.

\section{Conflicts of Interest}

The authors declare no conflicts of interest regarding the publication of this paper.

\section{References}

[1] Globocan 2018. https://www.uicc.org/news/new-global-cancer-data-globocan-2018 
[2] Matias, R., Fradique, C., Quaresma, L. and Aguiar, C. (2016) Gastric Cancer in Portugal: Where Do We Stand, East or West? Rev Port Cir Cardiotorac Vasc, 37, 21.

[3] Schlansky, B. and Sonnenberg, A. (2011) Epidemiology of Noncardia Gastric Adenocarcinoma in the United States. American Journal of Gastroenterology, 106, 1978-1985. https://doi.org/10.1038/ajg.2011.213

[4] Riihimäki, M., Hemminki, A., Sundquist, K., Sundquist, J. and Hemminki, K. (2016) Metastatic Spread in Patients with Gastric Cancer. Oncotarget, 7, 52307-52316. https://doi.org/10.18632/oncotarget.10740

[5] Kirchberger, M.C. (2018) Unusual Presentation of a Cutaneous Metastasis in the Face Arising from Gastric Cancer: A Case Report. SAGE Open Medical Case Reports, 6, 2050313X18795080. https://doi.org/10.1177/2050313X18795080

[6] Tso, S., Brockley, J., Recica, H. and Ilchyshyn, A. (2013) Sister Mary Joseph's Nodule: An Unusual but Important Physical Finding Characteristic of Widespread Internal Malignancy. British Journal of General Practice, 63, 551-552. https://doi.org/10.3399/bjgp13X673900

[7] Namikawa, T., Munekage, E., Munekage, M., et al. (2017) Subcutaneous Metastasis Arising from Gastric Cancer: A Case Report. Molecular and Clinical Oncology, 6, 515-516. https://doi.org/10.3892/mco.2017.1175

[8] Morais, S., Ferro, A., Bastos, A., Castro, C., Lunet, N. and Peleteiro, B. (2016) Trends in Gastric Cancer Mortality and in the Prevalence of Helicobacter Pylori Infection in Portugal. European Journal of Cancer Prevention, 25, 275-281.

https://doi.org/10.1097/CEJ.0000000000000183

[9] Castro, C., Peleteiro, B., Bento, M.J. and Lunet, N. (2017) Trends in Gastric and Esophageal Cancer Incidence in Northern Portugal (1994-2009) by Subsite and Histology, and Predictions for 2015. Tumori Journal, 103, 155-163. https://doi.org/10.5301/tj.5000542

[10] Hu, S.C., Chen, G.S., Wu, C.S., Chai, C.Y., Chen, W.T. and Lan, C.C. (2009) Rates of Cutaneous Metastases from Different Internal Malignancies: Experience from a Taiwanese Medical Center. Journal of the American Academy of Dermatology, 60, 379-387. https://doi.org/10.1016/j.jaad.2008.10.007

[11] Alcaraz, I., Cerroni, L., Rütten, A., Kutzner, H. and Requena, L. (2012) Cutaneous Metastases from Internal Malignancies: A Clinicopathologic and Immunohistochemical Review. The American Journal of Dermatopathology, 34, 347-393. https://doi.org/10.1097/DAD.0b013e31823069cf

[12] Namikawa, T. and Hanazaki, K. (2014) Clinicopathological Features and Treatment Outcomes of Metastatic Tumors in the Stomach. Surgery Today, 44, 1392-1399. https://doi.org/10.1007/s00595-013-0671-9

[13] Morlese, J.F., Jeswani, T., Beal, I., Wylie, P. and Bell, J. (2007) Acute Ventricular and Aortic Thrombosis Post Chemotherapy. The British Journal of Radiology, 80, e75-e77. https://doi.org/10.1259/bjr/26948582

[14] Apiyasawat, S., Wongpraparut, N., Jacobson, L., Berkowitz, H., Jacobs, L.E. and Kotler, M.N. (2003) Cisplatin Induced Localized Aortic Thrombus. Echocardiography, 20, 199-200. https://doi.org/10.1046/j.1540-8175.2003.03002.x

[15] Cheriparambil, K.M., Vasireddy, H., Kuruvilla, A., Gambarin, B., Makan, M. and Saul, B.I. (2000) Acute Reversible Cardiomyopathy and Thromboembolism After Cisplatin and 5-Fluorouracil Chemotherapy. Angiology, 51, 873-878.

https://doi.org/10.1177/000331970005101011

[16] Ikeda A., Yamachika, E., Mizutani, M., Matsubara, M., Moritani, N., Nakatsuji, K. and Iida, S. (2017) Rapid Occurrence of Left Ventricular Thrombus Associated with 
Platinum-Based Chemotherapy plus Cetuximab for the Treatment of Metastatic Squamous Cell Carcinoma of the Head and Neck: A Case Report. Molecular and Clinical Oncology, 7, 833-836. https://doi.org/10.3892/mco.2017.1393

[17] Kaul, P., George, R., Paniagua, R., Balaji, S., Sivananthan, M. and Sapsford, R. (2012) Massive Inferior Venacavo-Atrial Thrombus Following Neoadjuvant Chemotherapy. British Journal of Cardiology, 19, 184.

https://doi.org/10.5837/bjc.2012.034

[18] Yang, D., Hendifar, A., Lenz, C., et al. (2011) Survival of Metastatic Gastric Cancer: Significance of Age, Sex and Race/Ethnicity. Journal of Gastrointestinal Oncology, 2, 77-84.

[19] Bernards, N., Creemers, G.J., Nieuwenhuijzen, G.A., Bosscha, K., Pruijt, J.F. and Lemmens, V.E. (2013) No Improvement in Median Survival for Patients with Metastatic Gastric Cancer Despite Increased Use of Chemotherapy. Annals of Oncolo$g y$, 24, 3056-3060. https://doi.org/10.1093/annonc/mdt401

[20] Kanagavel, D., Pokataev, I.A., Fedyanin, M.Y., et al. (2010) A Prognostic Model in Patients Treated for Metastatic Gastric Cancer with Second-Line Chemotherapy. Annals of Oncology, 21, 1779-1785. https://doi.org/10.1093/annonc/mdq032 